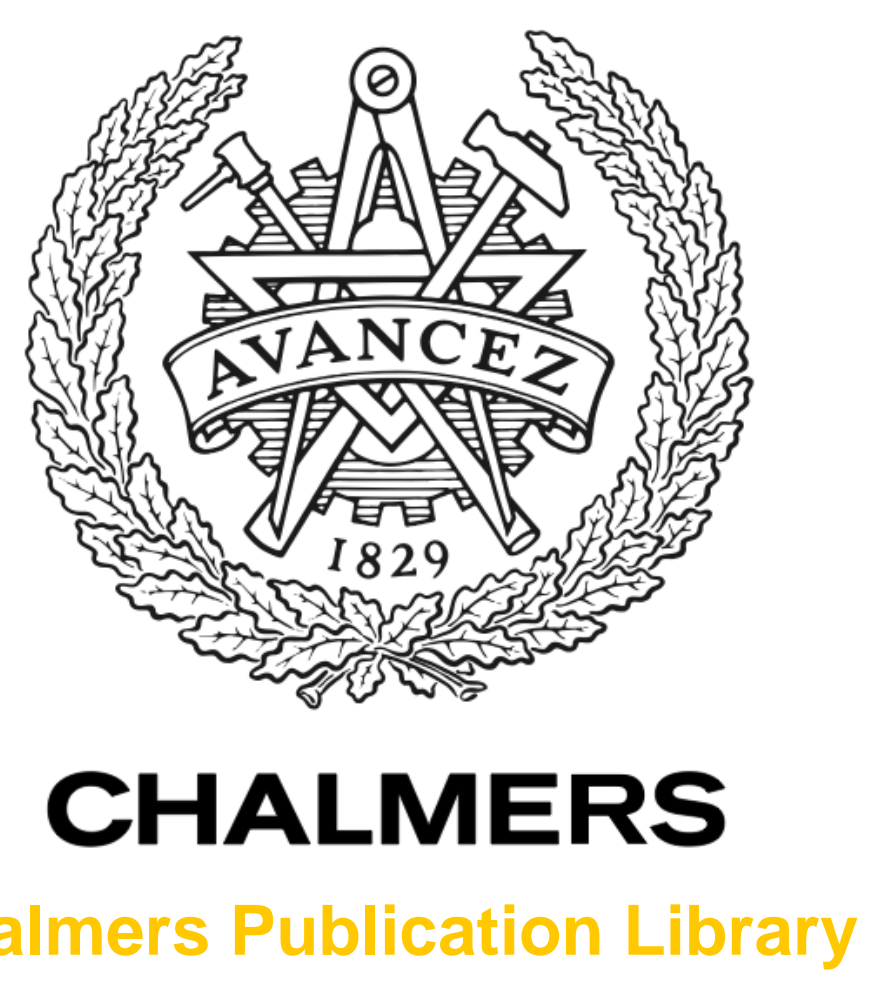

Chalmers Publication Library

A Simple Approximation for the Bit-Interleaved Coded Modulation Capacity

This document has been downloaded from Chalmers Publication Library (CPL). It is the author's version of a work that was accepted for publication in:

leee Communications Letters (ISSN: 1089-7798)

Citation for the published paper:

Alvarado, A. ; Brännström, F. ; Agrell, E. (2014) "A Simple Approximation for the Bit-

Interleaved Coded Modulation Capacity". Ieee Communications Letters, vol. 18(3), pp. 495-

498.

$\frac{\text { http://dx.doi.org/10.1109/lcomm.2014.011314.1326 }}{33}$

DownIoaded from: http://publications.lib.chalmers.se/publication/199410

Notice: Changes introduced as a result of publishing processes such as copy-editing and formatting may not be reflected in this document. For a definitive version of this work, please refer to the published source. Please note that access to the published version might require a subscription.

Chalmers Publication Library (CPL) offers the possibility of retrieving research publications produced at Chalmers University of Technology. It covers all types of publications: articles, dissertations, licentiate theses, masters theses, conference papers, reports etc. Since 2006 it is the official tool for Chalmers official publication statistics. To ensure that Chalmers research results are disseminated as widely as possible, an Open Access Policy has been adopted.

The CPL service is administrated and maintained by Chalmers Library. 


\title{
A Simple Approximation for the Bit-interleaved Coded Modulation Capacity
}

\author{
Alex Alvarado, Fredrik Brännström, and Erik Agrell
}

\begin{abstract}
The generalized mutual information (GMI) is an achievable rate for bit-interleaved coded modulation (BICM) and is highly dependent on the binary labeling of the constellation. The BICM-GMI, sometimes called the BICM capacity, can be evaluated numerically. This approach, however, becomes impractical when the number of constellation points and/or the constellation dimensionality grows, or when many different labelings are considered. A simple approximation for the BICMGMI based on the area theorem of the demapper's extrinsic information transfer (EXIT) function is proposed. Numerical results show the proposed approximation gives good estimates of the BICM-GMI for labelings with close to linear EXIT functions, which includes labelings of common interest, such as the natural binary code, binary reflected Gray code, etc. This approximation is used to optimize the binary labeling of the 32APSK constellation defined in the DVB-S2 standard. Gains of approximately $0.15 \mathrm{~dB}$ are obtained.
\end{abstract}

Index Terms-Binary labeling, bit-interleaved coded modulation, extrinsic information transfer, mutual information.

\section{INTRODUCTION AND MOTIVATION}

Bit-interleaved coded modulation (BICM) is a pragmatic approach for coded modulation (CM) [1]-[3]. The transmitter is constructed by serially concatenating a binary channel encoder, a bit-level interleaver, and a memoryless mapper. At the receiver, a suboptimal bit-wise decoder is used, where logarithmic likelihood ratios (LLRs) are calculated, deinterleaved, and passed to a soft-input binary decoder. Despite its suboptimality, BICM is very attractive from an implementation point of view because of its performance-flexibility tradeoff. BICM is used in most of the existing wireless standards, e.g., IEEE $802.11 \mathrm{a} / \mathrm{g} / \mathrm{h}$ and DVB-T2/S2/C2.

When the channel inputs are taken from a discrete constellation, the mutual information (MI) represents the largest achievable rate for a $\mathrm{CM}$ scheme with optimal decoding. The BICM generalized mutual information (BICM-GMI) [2], [3], sometimes called the BICM capacity, is an achievable rate for the (suboptimal) BICM decoder. The MI depends on the constellation, but the BICM-GMI also depends on the binary labeling. Both the MI and BICM-GMI can be evaluated numerically using, e.g., Gauss-Hermite $(\mathrm{GH})$ quadratures [4, Sec. III], Monte-Carlo integration [2], [5, Appendix A], histograms, or Riemann-sum integration. However, all these

Research supported by the European Community's Seventh's Framework Programme (FP7/2007-2013) under grant agreement No. 271986 and by the Swedish Research Council, Sweden (under grant \#621-2011-5950).

A. Alvarado is with the Dept. of Engineering, University of Cambridge, Cambridge CB2 1PZ, United Kingdom (email: alex.alvarado@ieee.org).

F. Brännström and E. Agrell are with the Dept. of Signals and Systems, Chalmers Univ. of Technology, SE-41296 Göteborg, Sweden (email: $\{$ fredrik.brannstrom, agrell $\} @$ chalmers.se). methods typically become impractical when the number of constellation points and/or the number of dimensions grow.

The BICM-GMI can be optimized for a given constellation by properly selecting the binary labeling. Analytical results are available for low and high signal-to-noise ratios (SNRs) [6], [7], but for finite SNR values, numerical optimization methods need to be applied. One of the most popular algorithms is the binary switching algorithm (BSA) [8, Sec. IV], popularized in [9] in the context of BICM with iterative demapping. The BSA iteratively improves a given initial labeling evaluating a cost function at each step. In [9], the Chernoff bound on the pairwise error probability was used as a low complexity cost function. A simulated annealing algorithm was recently proposed in [10], [11], where the labeling and the constellation are jointly optimized. Although the approaches in [10], [11] used the BICM-GMI as a cost function (calculated using GH quadratures), simple approximations for the BICM-GMI would simplify the optimization.

The main contribution of this letter is a simple approximation for the BICM-GMI. The approximation is based on the area theorem [12, Sec. IV-A] applied to the extrinsic information transfer (EXIT) function of the demapper [3, Sec. 5.4], which is approximated by a straight line, combined with an approximation of the so-called $J$-function [13, Sec. III]. Numerical results show that the proposed approximation predicts the true BICM-GMI well in many cases. It seems particularly well suited to some particular labelings such as Gray codes.

\section{System Model}

We consider a discrete-time, real-valued $N$-dimensional additive white Gaussian noise (AWGN) channel $\boldsymbol{Y}=\boldsymbol{X}+\boldsymbol{Z}$, where the noise $\boldsymbol{Z}$ is an $N$-dimensional Gaussian random vector, independent of the input $\boldsymbol{X}$, whose components are independent and identically distributed (i.i.d.) Gaussian random variables with zero mean and variance $N_{0} / 2$. The capacity of this channel is given by Shannon's formula $C^{\text {aw }} \triangleq$ $\frac{N}{2} \log _{2}\left(1+\frac{2 \gamma}{N}\right)$, where $\gamma \triangleq \frac{E_{\mathrm{s}}}{N_{0}}$ and $E_{\mathrm{s}} \triangleq \mathbb{E}\left[\|\boldsymbol{X}\|^{2}\right]=1$.

The transmitted symbols $\boldsymbol{X}$ are drawn uniformly from a constellation $\mathcal{X} \triangleq\left\{\boldsymbol{x}_{1}, \ldots, \boldsymbol{x}_{M}\right\}$, where $|\mathcal{X}|=M=2^{m}$. The distinct nonzero Euclidean distances in the constellation are denoted by $\boldsymbol{d}=\left[d_{1}, \ldots, d_{L}\right]$, with $d_{l}<d_{l+1}, l=1, \ldots, L-1$. The number of pairs of constellation points at Euclidean distance $d_{l}$ whose labels are at Hamming distance one is denoted by $w_{l}$. Since each symbol has $m$ symbols at Hamming distance one, the sum of the elements in $\boldsymbol{w} \triangleq\left[w_{1}, \ldots, w_{L}\right]$ is $m M / 2$.

The system model is shown in Fig. 1, where a binary encoder of rate $R_{\mathrm{c}}$ and a bit-level interleaver are concatenated 


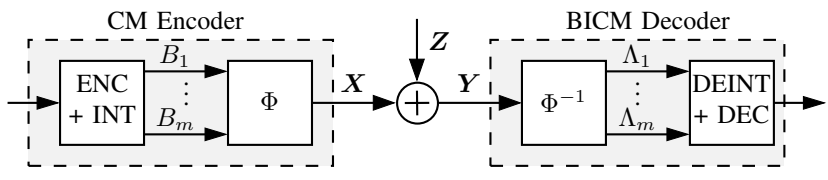

Fig. 1. BICM system model under consideration.

with a memoryless mapper $\Phi$. At the receiver side, LLRs $\Lambda_{k}$, $k=1, \ldots, m$ are calculated and passed to a deinterleaver and then to a soft-input binary decoder. When communicating at rate $R=m R_{\mathrm{c}}$, the average bit energy is $E_{\mathrm{b}}=E_{\mathrm{s}} / R$.

The CM mutual information (CM-MI) is defined as the input-output MI of the channel [14, eq. (5)], i.e., $I^{\mathrm{cm}} \triangleq$ $I(\boldsymbol{X} ; \boldsymbol{Y})$. The BICM-GMI is defined as [14, eq. (10)]

$$
I^{\mathrm{bi}} \triangleq \sum_{k=1}^{m} I\left(B_{k} ; \boldsymbol{Y}\right) \leq I^{\mathrm{cm}},
$$

where $I\left(B_{k} ; \boldsymbol{Y}\right)$ is the MI between $B_{k}$ and $\boldsymbol{Y}$, and $B_{k}$ are the input bits to the memoryless mapper. The bits $B_{k}$ are assumed to be i.i.d. random variables with $\operatorname{Pr}\left\{B_{k}=b\right\}=1 / 2$ for $k=1, \ldots, m$ and $b \in\{0,1\}$. The BICM-GMI in (1) was shown to be an achievable rate in [14], where the BICM decoder in Fig. 1 was cast as a mismatched decoder.

\section{MAIN RESULT}

Let $I^{\operatorname{dem}}(i) \in[0,1]$ be the demapper's EXIT function [13], [15], where the a priori information $i \in[0,1]$ is modeled using a binary erasure channel (BEC) [12, Sec. IV-A], [3, Sec. 5.3]. The area theorem [3, Corollary 5.2] states that

$$
\int_{0}^{1} I^{\mathrm{dem}}(i) \mathrm{d} i=\frac{1}{m} I^{\mathrm{cm}} .
$$

The zero a priori information value can be shown to be a scaled version of the BICM-GMI [3, Sec. 5.3], [15, eq. (65)]

$$
I^{\mathrm{dem}}(0)=I^{\mathrm{bi}} / m \text {. }
$$

The full a priori value is [15, eq. (60)]

$$
I^{\mathrm{dem}}(1)=\frac{2}{m M} \sum_{l=1}^{L} w_{l} J\left(d_{l} \sqrt{2 \gamma}\right),
$$

where $J(\cdot)$ is the so-called $J$-function [13, eq. (7)], which represents the MI between a pair of constellations points at a given Euclidean distance. Note that both $I^{\mathrm{dem}}(0)$ and $I^{\mathrm{dem}}(1)$ are independent of the BEC model used in (2).

The main result of this letter is the following approximation for the BICM-GMI in (1):

$$
I^{\mathrm{bi}} \approx \tilde{I}^{\mathrm{bi}} \triangleq 2 I^{\mathrm{cm}}-\frac{2}{M} \sum_{l=1}^{L} w_{l} \tilde{J}\left(d_{l} \sqrt{2 \gamma}\right),
$$

where [13, eq. (9)]

$$
J(x) \approx \tilde{J}(x) \triangleq\left(1-2^{-0.3073 x^{1.7870}}\right)^{1.1064} .
$$

The approximation in (5) follows by considering a linear

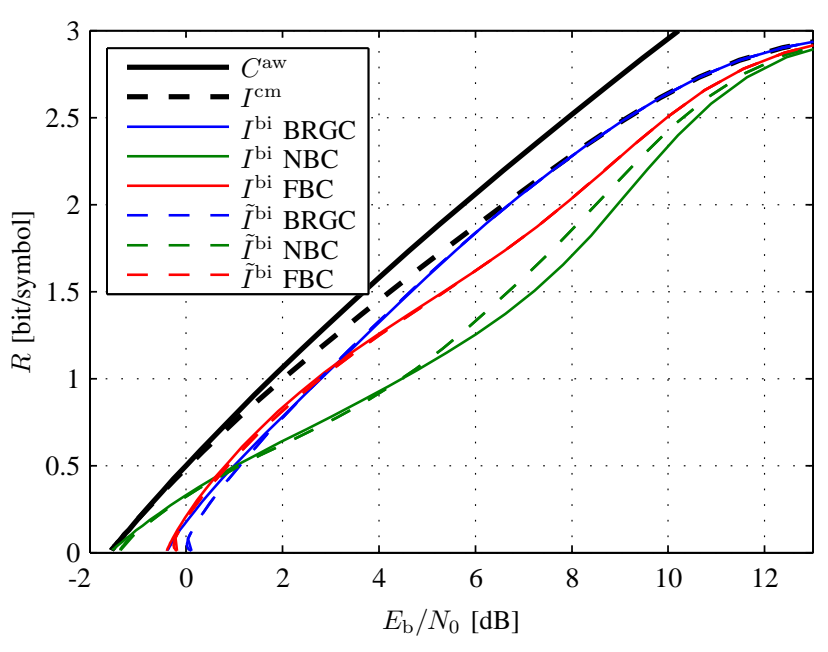

Fig. 2. The CM-MI, the exact BICM-GMIs (solid lines) and the approximation in (5) (dashed lines) for an 8-PAM constellation.

approximation of the demapper's EXIT function ${ }^{1}$

$$
I^{\mathrm{dem}}(i) \approx \tilde{I}^{\mathrm{dem}}(i) \triangleq I^{\mathrm{dem}}(0)+i\left(I^{\mathrm{dem}}(1)-I^{\mathrm{dem}}(0)\right)
$$

Combining (7) with (2), we obtain

$$
\frac{1}{m} I^{\mathrm{cm}} \approx \int_{0}^{1} I^{\mathrm{dem}}(0)+i\left(I^{\mathrm{dem}}(1)-I^{\mathrm{dem}}(0)\right) \mathrm{d} i,
$$

which results in

$$
m I^{\mathrm{dem}}(0) \approx 2 I^{\mathrm{cm}}-m I^{\mathrm{dem}}(1) .
$$

The expression in (5) is obtained by using (3) and (4) in (9), and by using the approximation of the $J$-function in (6).

\section{NUMERICAL EXAMPLES}

The approximation in (5) allows us to approximate the BICM-GMI for any given constellation and binary labeling. Consider an equally spaced 8 -ary pulse amplitude modulation (PAM) constellation $\left(d_{l}=2 l / \sqrt{21}, l=1, \ldots, 7\right)$ and the three labelings in [6, Example 1]: the binary reflected Gray code (BRGC) with $\boldsymbol{w}=[7,0,3,0,1,0,1]$, the natural binary code (NBC) with $\boldsymbol{w}=[4,4,0,4,0,0,0]$, and the folded binary code (FBC) with $\boldsymbol{w}=[5,4,1,0,1,0,1]$. In Fig. 2, we show the BICM-GMIs for these three labelings (numerically calculated using $50 \mathrm{GH}$ quadratures [4, Sec. III]) as well as the approximation in (5). The CM-MI and the channel capacity are also included for comparison. The results in Fig. 2 show that the proposed approximation works well for the BRGC and FBC for the entire SNR range of interest. For the NBC, the approximation is not tight for medium SNRs. However, it still gives good results for high and low SNRs.

As (5) is an approximation, some discrepancies are indeed observed in Fig. 2. The first approximation comes from considering a linear model for the EXIT function of the demapper in (7). This model is relatively good for labelings with close-to-linear EXIT functions. These are the labelings

\footnotetext{
${ }^{1}$ The EXIT function of the demapper can be shown to be a polynomial function of $i$ with degree $m-1$, see, e.g., [16, eqs. (10), (15), and (19)].
} 


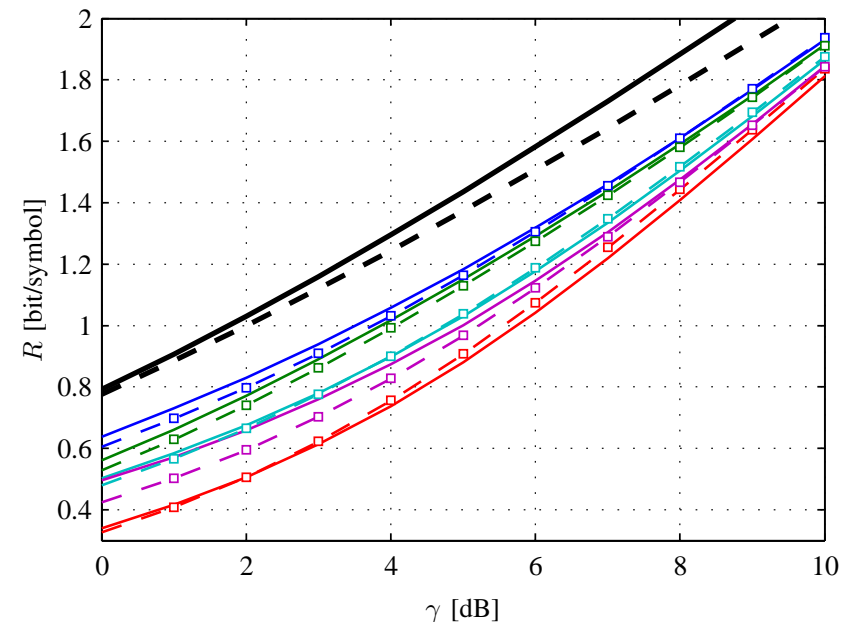

Fig. 3. The CM-MI, the exact BICM-GMIs (solid lines) and the approximation in (5) (dashed lines) for a 16-PAM constellation and 5 randomly chosen Gray codes. The squares show the approximation in (5) using the $J$-function computed via $\mathrm{GH}$ quadratures.

usually analyzed in the literature: Gray or quasi-Gray codes [16, Fig. 2], set-partitioning [3, Fig. 5.8 (a)], the natural binary code [15, Fig. 8], etc. The second approximation comes from considering an easy-to-evaluate approximation for the $J$-function in (6). When compared to the $J$-function computed using $50 \mathrm{GH}$ quadratures, we have verified that the approximation (6) results in errors always smaller than $10^{-3}$.

Consider now an equally spaced 16-PAM constellation $\left(d_{l}=2 l / \sqrt{85}, l=1, \ldots, 15\right)$. For this constellation the number of different labelings is very large, so we focus only on Gray codes. For 16-PAM, there are 131 nonequivalent Gray codes in terms of uncoded bit error rate (BER) [17, Table I], from which we randomly chose five. The BICMGMIs for those labelings are shown in Fig. 3 together with their approximations in (5). These results show a good match between the true and approximated BICM-GMI. More importantly, the results in this figure also show that, for a given SNR value, a maximization of the true BICM-GMI is equivalent to a maximization of the approximated BICM-GMI.

In Fig. 3, we also show the results obtained when the $J$ function is computed using GH quadratures, which shows that the use of the approximation of the $J$-function in (6) is indeed very tight. To study the tightness of the proposed approximation, we introduce the error function $\varepsilon \triangleq I^{\mathrm{bi}}-\tilde{I}^{\mathrm{bi}}$. Since $\tilde{J}(x)=J(x)$ as $x \rightarrow 0$ or $x \rightarrow \infty$, we conclude that $\varepsilon=0$ as $\gamma \rightarrow 0$ and $\gamma \rightarrow \infty$, i.e., the approximation is tight for both high and low SNR. In Fig. 4, we show the error $\varepsilon$ for the same binary labelings as in Fig. 3 when the $J$ function is evaluated using GH quadratures or via (6). These results show that $\varepsilon$ tends to zero at low and high SNR, and that $\varepsilon$ is in general very small for different labelings across the SNR range. This figure also shows that the error can be both positive and negative, and thus, the approximation $\tilde{I}^{\text {bi }}$ is neither an upper nor a lower bound to $I^{\mathrm{bi}}$, which is also clear from Figs. 2 and 3. More importantly, Fig. 4 also shows that the use of (6) results in no noticeable difference.

Lastly, we consider a 32-ary amplitude phase shift keying

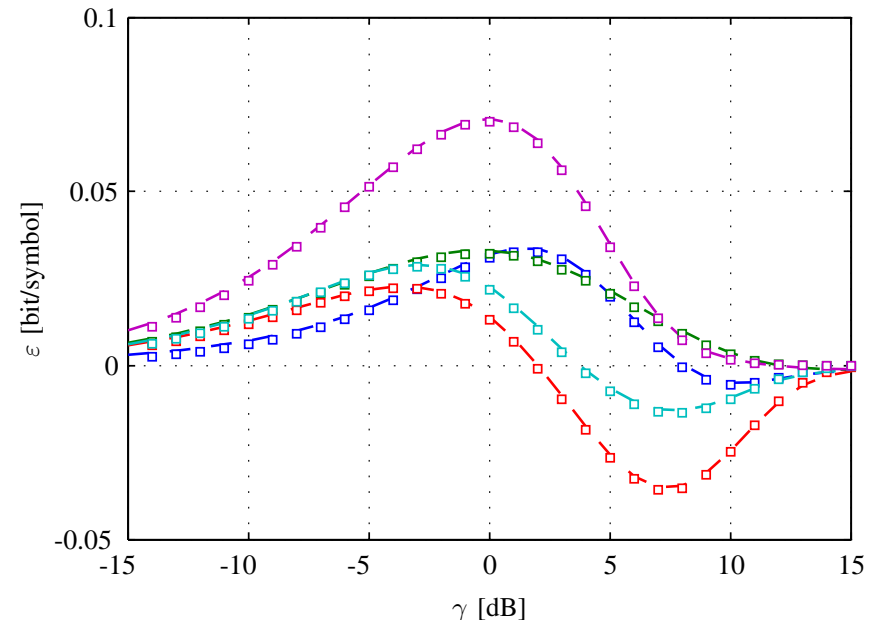

Fig. 4. The error $\varepsilon=I^{\text {bi }}-\tilde{I}^{\text {bi }}$ for the binary labelings in Fig. 3. The dashed lines are for the approximation in (5), while the squares are when the $J$-function is computed via GH quadratures.

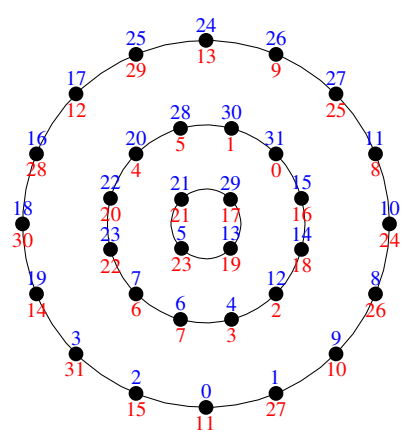

Fig. 5. 32-APSK constellation with the DVB-S2 labeling (red) and the new labeling found numerically using the approximation in (5) (blue).

(APSK) constellation defined in the DVB-S2 standard [18, Fig. 13] where the values of the three radii depend on the targeted spectral efficiency $R$ [18, Table 10]. Here we consider $R_{\mathrm{c}}=$ $3 / 4(R=3.75$ bit/symbol with $L=57$ distinct Euclidean distances), and thus, $\boldsymbol{d}=[0.34,0.36,0.45,0.48,0.49,0.50, \ldots]$. The 32-APSK constellation is shown in Fig. 5, where the binary labeling proposed in the DVB-S2 standard (from now on called "DVB-S2 labeling") is shown (in decimal) below the constellation points (in red). For this labeling, $\boldsymbol{w}=[4,12,0,0,8,12, \ldots]$. The corresponding BICM-GMI and its approximation are shown in Fig. 6 (in red). Again, our approximation predicts the BICM-GMI well for the SNR range of interest.

To prove the usefulness of our approximation, we implemented the BSA using the sum in the right-hand side of (5) as cost function and ran it for the constellation in Fig. 5 for $\gamma=12 \mathrm{~dB}$. The best labeling found, which is shown in Fig. 5 above the constellation points, gives a higher BICM-GMI than the DVB-S2 labeling, and has $\boldsymbol{w}=[4,12,4,0,0,16, \ldots]$. The corresponding BICM-GMI and its approximation are shown in Fig. 6 (blue), where for rates around 3.75 bit/symbol, gains of $0.15 \mathrm{~dB}$ are observed. Similar gains have been recently reported in [11]. Although not a large gain, the results demonstrate the usefulness of the approximation. 


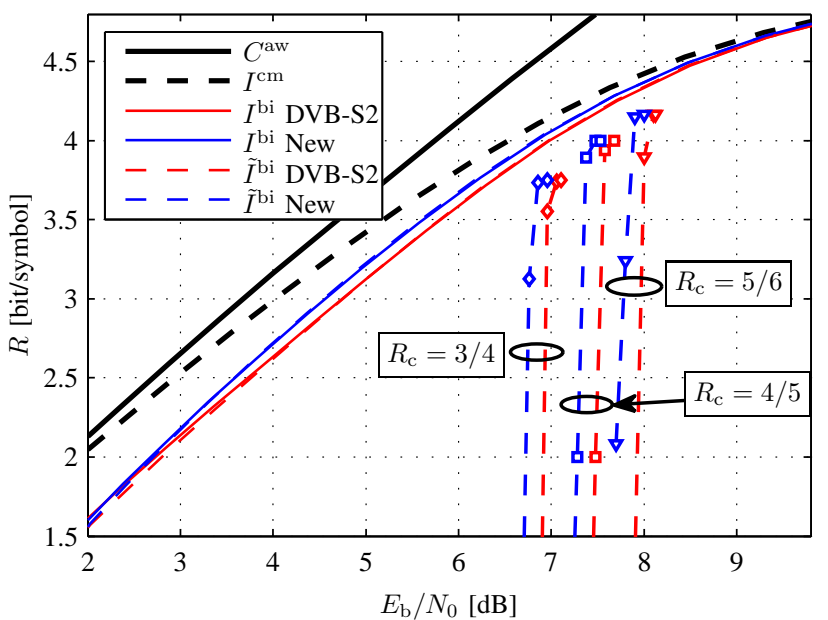

Fig. 6. The CM-MI, the exact BICM-GMIs (solid lines) and the approximation in (5) (dashed lines) for the 32-APSK constellation in the DVB-S2 standard. The BICM-GMI for the new labeling is also shown. The throughputs $\Theta$ based on the FERs in Fig. 7 are also included (dashed lines)

To conclude, we simulate the low-density parity-check (LDPC) code proposed in the DVB-S2 standard (without the $\mathrm{BCH}$ outer code) for frames of 12960 APSK symbols with the new binary labeling. We use the belief propagation algorithm and 100 iterations for decoding. For simplicity, we consider the constellation and labelings in Fig. 5 together with the three code rates: $R_{\mathrm{c}} \in\{3 / 4,4 / 5,5 / 6\}$ (instead of three different constellations and possibly three new labelings). The BER and frame error rate (FER) results in Fig. 7 show that the gains in terms of BICM-GMI translate into BER/FER performance. Finally, assuming frames in error are perfectly detected at the receiver, we consider the effective throughput $\Theta \triangleq m R_{\mathrm{c}}(1-P)[19$, eq. (4)], where $P$ denotes the FER shown in Fig. 7. The corresponding throughputs are shown in Fig. 6. These results indicate that the "envelope" of the throughput curves follow the BICM-GMI curves (as previously shown in $[19$, Sec. V]), and that the gap between the LDPCcoded system and the BICM-GMI is approximately $0.6 \mathrm{~dB}$.

\section{CONCLUSiOnS}

A simple approximation for the BICM-GMI was presented. It depends on the MI of the underlying constellation and the extrinsic information produced by the demapper when full a priori information is available. Due to its simplicity, it allows fast numerical estimation of the BICM-GMI for a given constellation and different labelings, which, as demonstrated by examples, can help improve BICM system designs. Future work includes a refined model for the approximation of the EXIT function in (7) and a formal analysis of the error.

\section{REFERENCES}

[1] E. Zehavi, "8-PSK trellis codes for a Rayleigh channel," IEEE Trans. Commun., vol. 40, no. 3, pp. 873-884, May 1992.

[2] G. Caire, G. Taricco, and E. Biglieri, "Bit-interleaved coded modulation," IEEE Trans. Inf. Theory, vol. 44, no. 3, pp. 927-946, May 1998.

[3] A. Guillén i Fàbregas, A. Martinez, and G. Caire, "Bit-interleaved coded modulation," Foundations and Trends in Communications and Information Theory, vol. 5, no. 1-2, pp. 1-153, 2008.

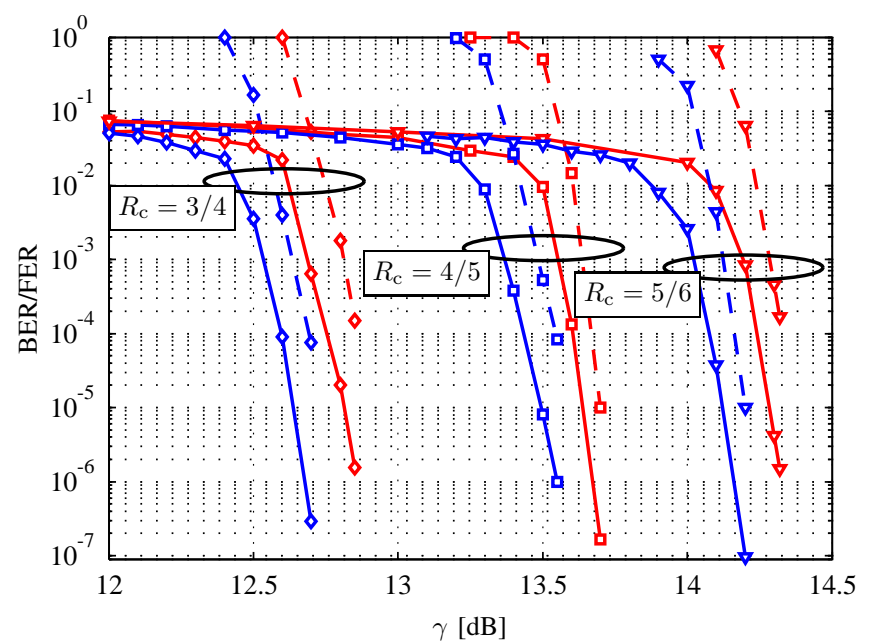

Fig. 7. BER (solid lines) and FER (dashed lines) for the 32-APSK constellation with the DVB-S2 (red) and the new labeling in Fig. 5 (blue)

[4] A. Alvarado, F. Brännström, and E. Agrell, "High SNR bounds for the BICM capacity," in IEEE Information Theory Workshop (ITW), Paraty, Brazil, Oct. 2011.

[5] P. Fertl, J. Jaldén, and G. Matz, "Performance assessment of MIMOBICM demodulators based on system capacity," IEEE Trans. Signal Process., vol. 60, no. 3, pp. 2764-2772, Mar. 2012.

[6] E. Agrell and A. Alvarado, "Optimal alphabets and binary labelings for BICM at low SNR," IEEE Trans. Inf. Theory, vol. 57, no. 10, pp. 6650-6672, Oct. 2011.

[7] A. Alvarado, F. Brännström, E. Agrell, and T. Koch, "High-SNR asymptotics of the mutual information with applications to BICM," IEEE Trans. Inf. Theory (to appear), 2014.

[8] K. Zeger and A. Gersho, "Pseudo-Gray coding," IEEE Trans. Commun., vol. 38, no. 12, pp. 2147-2158, Dec. 1990.

[9] F. Schreckenbach, N. Görtz, J. Hagenauer, and G. Bauch, "Optimization of symbol mappings for bit-interleaved coded modulation with iterative decoding," IEEE Commun. Lett., vol. 7, no. 12, pp. 593-595, Dec. 2003.

[10] F. Kayhan and G. Montorsi, "Joint signal-labeling optimization for pragmatic capacity under peak-power constraint," in IEEE Global Telecommunications Conference (GLOBECOM), Miami, FL, Dec. 2010.

[11] _ " "Constellation design for transmission over nonlinear satellite channels," in IEEE Global Telecommunications Conference (GLOBECOM), Anaheim, CA, Dec. 2012.

[12] A. Ashikhmin, G. Kramer, and S. ten Brink, "Extrinsic information transfer functions: Model and erasure channel properties," IEEE Trans. Inf. Theory, vol. 50, no. 11, pp. 2657-2673, Nov. 2004.

[13] F. Brännström, L. K. Rasmussen, and A. J. Grant, "Convergence analysis and optimal scheduling for multiple concatenated codes," IEEE Trans. Inf. Theory, vol. 51, no. 9, pp. 3354-3364, Sep. 2005.

[14] A. Martinez, A. Guillén i Fàbregas, and G. Caire, "Bit-interleaved coded modulation revisited: A mismatched decoding perspective," IEEE Trans. Inf. Theory, vol. 55, no. 6, pp. 2756-2765, June 2009.

[15] F. Brännström and L. K. Rasmussen, "Classification of unique mappings for 8PSK based on bit-wise distance spectra," IEEE Trans. Inf. Theory, vol. 55, no. 3, pp. 1131-1145, Mar. 2009.

[16] W. Carson, M. Rodrigues, and I. Wassell, "Characterization of demapper EXIT functions with BEC a priori information with applications to BICM-ID," IEEE Trans. Commun., vol. 59, no. 11, pp. 3080-3089, Nov. 2011.

[17] E. Agrell, J. Lassing, E. G. Ström, and T. Ottosson, "On the optimality of the binary reflected Gray code," IEEE Trans. Inf. Theory, vol. 50, no. 12, pp. 3170-3182, Dec. 2004.

[18] ETSI, "Digital video broadcasting (DVB); Second generation framing structure, channel coding and modulation systems for broadcasting, interactive services, news gathering and other broadband satellite applications (DVB-S2)," ETSI, Tech. Rep. ETSI EN 302307 V1.2.1 (200908), Aug. 2009.

[19] A. Alvarado, H. Carrasco, and R. Feick, "On adaptive BICM with finite block-length and simplified metrics calculation," in IEEE Vehicular Technology Conference (VTC-Fall), Montreal, QC, Canada, Sep. 2006. 